\title{
Could Nanoparticles Reinforce Polymer Matrices?
}

\author{
G. Schrodj, M.-P. Hirn, B. Haidar* \\ Institut de Science de Materiaux de Mulhouse, CNRS-UMR 7361, Mulhouse, France \\ Email: *bassel.haidar@uha.fr
}

Received August 2013

\begin{abstract}
In this work, we challenge the idea that "introducing nanoparticles in polymer matrices enhance properties" which is assumed spreading almost dogmatically. Two series of compounds were prepared: one based on elastomers (solution-SBR) filled with conventional carbon black particles, CB, the other used the same polymer but filled with carbon nanotubes, CNT. The results of two types of experiments were compared on the two series. The first is physical based on the non linear response of filled materials to static deformation, the second physicochemical, stands on calorimetric measurements of the polymer heat of adsorption on the solid. Static deformation effect on dynamic mechanical modulus shows the behavior of the CB filled elastomers was qualitatively identical to that of glassy polymer reflecting the existence of an immobilized fraction of the polymer at the intephase, while that of CNT was found identical to unfilled polymer indicating a poor filler-polymer interactions. Polymer adsorption measured by flow micro calorimeter showed a substantial amount of heat exchange on the surface of CB while no heat of adsorption was detected on CNT. The lack of interaction between the polymer and the CNT, except for a small domain of a narrow polymer molecular weight, prevent any enhancements of mechanical properties. Other applications may be improved.
\end{abstract}

Keywords: Polymer; Nanoparticle; Dynamic Modulus; Static Deformation; Adsorption; Calorimeter; Interphase

\section{Introduction}

Expectations of nanoparticles benefits in polymer based materials rose out of proportion, as it is very often the case when a new technology immerges. All kind of fortunes, and miss fortunes, were attributed to the nanoaspect of this kind of particles: reinforcing and toughening effects; electrical, thermal and acoustical virtues; transport properties optical and aging enhancements... [1] However, when useful real products were late to the market or appeared less exciting than what it had been announced, expectations fell as rapidly as it had descended to an overwhelming low level. Only then, thoughts go back to basics: chemistry, physics and polymer science. We most likely are for the time being at this stage of nanoparticles development in polymer science.

For instance, the driving force behind any effect of solid particles on the behavior of polymer matrix is the way polymer chains get in contact with the solid surface. Adsorption, affinity, interactions, immobilization, interphase buildup... are only a few of many concepts developed in the literature to emphasize the importance of polymer/filler contact-adjustment on the properties and behaviors of the compound.

In this work, we tempted to compare the effect if particles size-scale on two of the polymers prominent prop-

*Corresponding author. erties: Dynamic-mechanical and physicochemical.

\section{Nonlinear Behavior of Filled Polymer as Detected by Dynamic Mechanical Behavior}

Nonlinear response of polymer material do deformation can be demonstrated by different means. One of the most elegant is that of TL. Smith [2] which consists of applying at a time, $\mathrm{t}_{0}$, à constant macroscopic strain, $\lambda$, on the polymer specimen (as in relaxation test) and superimposes every now and then on $\lambda$ a dynamic deformation of constant frequency, $f$, and amplitude, $\varepsilon$, small enough be considered as affiliated to the linear deformation.

\subsection{Behavior of Glassy Polymers}

In the case of glassy polymers such as polycarbonate, Poly (methyl methacrylate), polystyrene... dynamic modulus, e.g. E' schematically presented in Figure 1, decreases suddenly upon deformation from its original value, $E_{0}{ }_{0}$, to an undetectable level. Results show linear modulus-time dependency in a log-log scale, the slope of which is $\mu$. This behavior was attributed to the nonequilibrium stat of glassy polymers, the so called "physical aging” phenomenon and its consequences as a decrease of the modulus (increase of the polymer segmental mobility, “des-aging”) upon deformation thereafter, and 


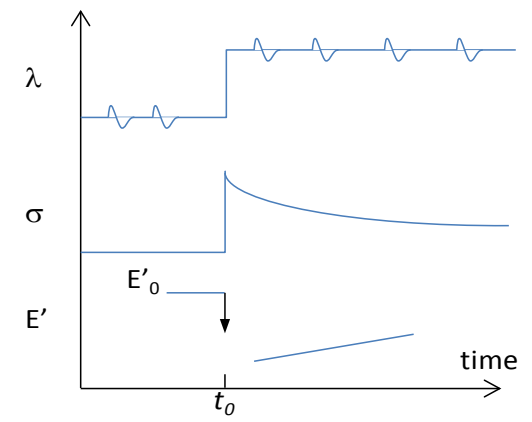

Figure 1. Schematic presentation of the dynamic mechanical experiment.

under relaxation conditions, a continues increase of the modulus because of the decrease of segmental mobility, "aging” anew. $\mu$ therefore is considered as an assessment of the physical aging rate.

\subsection{Behavior of Elastomers}

A solution-SBR specimen was crosslinked using 2 phr of dicumil peroxide. The effect of a static deformation, $\lambda=$ $1 / \mathrm{l}_{0}=1.03$ on dynamic modulus when tested at $-100^{\circ} \mathrm{C}, \mathrm{T}$ $<\mathrm{Tg}$, de-aging followed by aging processes were clearly observed, Figure 2. In contrast to the glassy state, the high segmental mobility of the polymer at the rubbery state places it in a quasi-thermodynamic equilibrium. Therefore measurements made at $30^{\circ} \mathrm{C}$ under similar relaxation conditions, the modulus $\mathrm{E}^{\prime}$ of the same crosslinked elastomers is virtually unaffected by a static deformation, at least as long as such a deformation remains moderate as shown also in Figure 2.

\subsection{Behavior of Polymer Filled with Conventional Particles}

The same solution-SBR was filled with 45 phr carbon black N-330 before crosslinking.

CB filled SBR showed a clear de-aging followed by aging processes, just as the unfilled polymer behaves at the glassy state, Figure 2. This behavior was attributed to the presence, in filled rubber, of a rubber/filler interfacial region with a specific behavior [3]. The polymer within this "interphase" has a low segmental mobility compared to the free rubber matrix and present, therefore, a glassy like behavior. Such interphase results most likely form the interactions that polymer most exchange with the solid surface. Such a specifically low segmental behavior of the rubber at the filler interface was already detected especially by ${ }^{1} \mathrm{H}$ solid NMR measurements [4].

\subsection{Behavior of Polymer Filled with Nanoparticles}

When filler particles are replaced in SBR based matrix

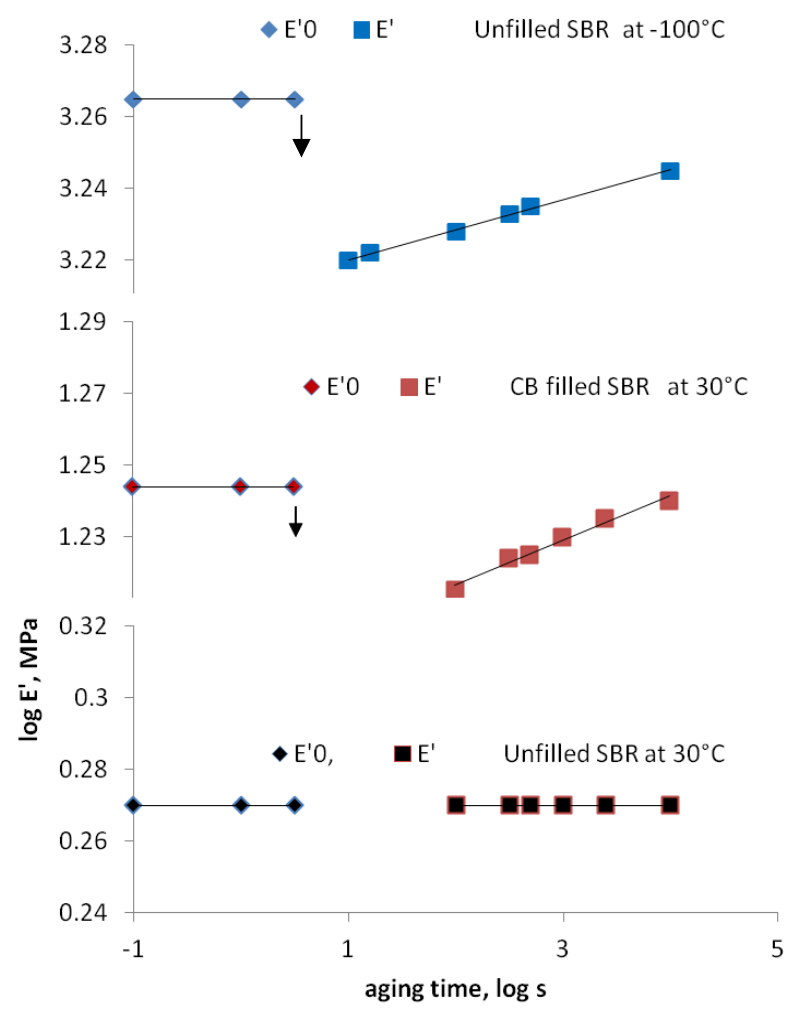

Figure 2. E' before, E'0, and after static deformation for different compounds précised on the graph.

by nanoparticles (carbon nanotubes, diameter 25 - $12 \mathrm{~nm}$, 1 - $2 \mu$ length), while all other conditions are kept identical to the ones adopted for conventional filler, we obtain the same behavior as for unfilled polymer, Figure 3. This may be a strong indication that no interphase is created at the nano tubes/polymer interface which presumably due to a lack of interactions exchange between the two actors. Under these circumstances one would not expect a substantial "reinforcing” effect beyond the hydrodynamic resulting from the filler addition which is noticed by the increase of the modulus compare Figures 2 and 3.

\section{Calorimetric Evaluation of Polymer-Filler Interactions}

Previous results stress the need for a direct measurement of filler-polymer interactions. Calorimetric approach using a flow calorimeter appeared as well adapted to achieve this goal.

A Flow Micro Calorimeter (FMC) was used to measure the polymer solution heat of adsorption on the filler. It operated under a constant flow rate $(3.3 \mathrm{ml} / \mathrm{h})$ of carrier solvent, $\mathrm{THF}$, at $25^{\circ} \mathrm{C}$. In a pulse mode, a known amount of the polymer dissolved in the carrier solvent was injected in the flow path through a calibrated sample loop. If any thermal exchange occurs when the solute 
comes into contact with the particles being studied, a peak was registered (positive or negative corresponding to exo- or endothermic events respectively, as schematized in Figure 4). The unadsorbed or/and desorbed fraction of the solute, if any, was then detected by a downstream detector. The polymer concentration was fixed at $10 \mathrm{~g} / \mathrm{l}$. The FMC principles and the experimental setup were described elsewhere [40-43].

\subsection{Heat of SSBR Adsorption on CNT and CB}

The peaks of adsorption of a solution of SSBR of CB and CNT areas presented in Figure 5.

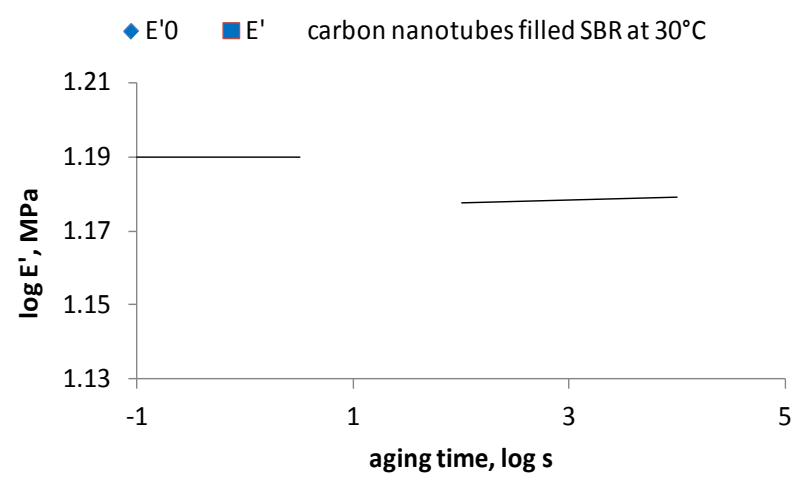

Figure 3. Effect of static deformation on E' for CNT filled SSBR.

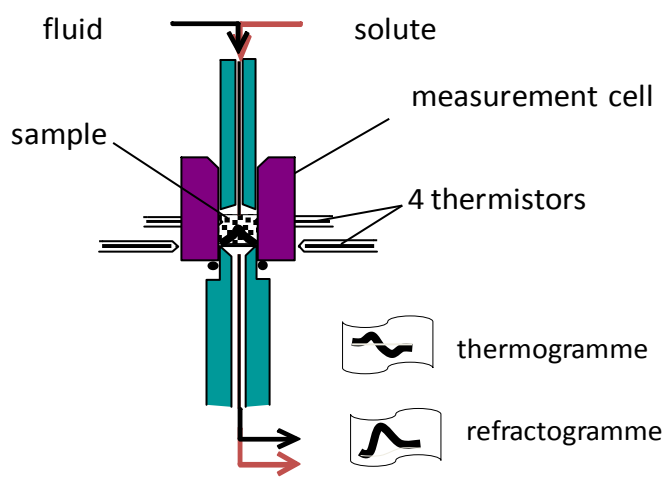

Figure 1. Schematic presentation of FMC.

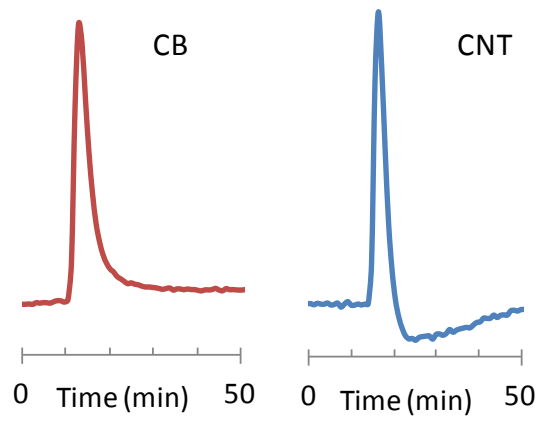

Figure 5. FMC thermogrammes of SSBR solution on CB and CNT.
In the case of CB we observe an exothermic peak indicating an irreversible adsorption resulting from a clear interaction exchanged between the polymer and the solid surface, while in the case of CNT, although a similar exothermic peak appears first, it is followed right after by an equivalent endothermic one reflecting reversible adsorption or two successive processes of adsorption and desorption; the final balance of the two processes is quite close to zero while for CB the balance is largely exothermic. The estimation of the molar heat of adsorption on $\mathrm{CB}$ gives a value in the range of chemical bounding, several hundreds of $\mathrm{kJ} / \mathrm{mol}$ which may explain the irreversible aspect of the adsorption in this case.

\subsection{The Effect of the Molecular Weight of Polybutadien on Its Heat of Adsorption on CNT}

The absence of a permanent adsorption on CNT in contrast with $\mathrm{CB}$ is unexpected and requires more investigation. The most important difference between $\mathrm{CB}$ and CNT is their forms: aspect ratio and size scale; although chemical defects and edges of graphite plan are more prominent in $\mathrm{CB}$ than in $\mathrm{CNT}$, both solid surfaces are essentially formed out graphite plans. Under theses circumstances it was legitimate to question the reticence of CNT to adsorb polymer on the base of scale size matching of the two substances. In fact, the size of a CB aggregate is quite huge compared to CNT diameter, which approaches the radius of gyration of a long polymer chain itself. The behavior of the polymer chain toward the two kinds of surfaces might be quite different.

Therefore, we synthesized a series of polybutadienes with identical micro-structure (1,2-PB content equal to about $80 \%$, same Tg), various molecular weights (from thousand to several hundred-thousand g/mol) and a very narrow molecular weight distribution $\left(\mathrm{I}_{\mathrm{p}} \approx 1.1\right)$. Results obtained by the application of FMC experimental approach on CNT are show in Figure 6.

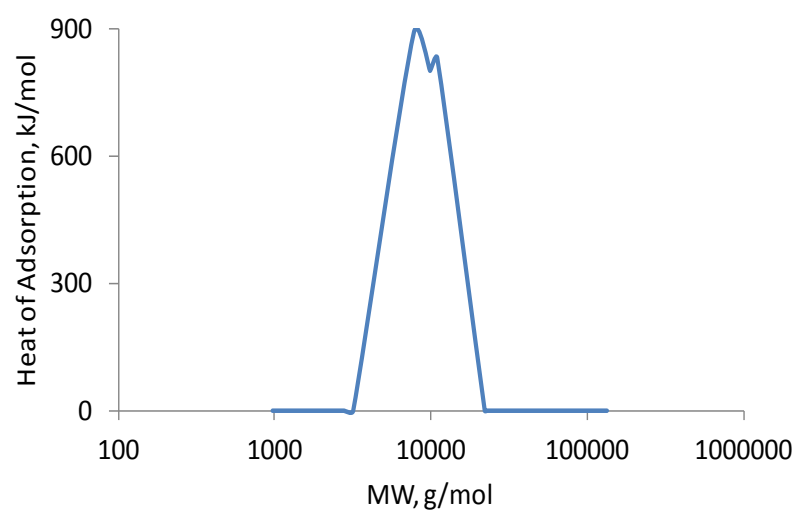

Figure 6. Molar heat of adsorption as a function of molecular weight of polybutadiene. 
It is clear that for linear amorphous polymer adsorption takes place exclusively in a relatively small window of MW's. Adsorption, within this window, is irreversible and associated with substantial amount of heat, whereas outside its limits adsorption is reversible and heat exchange is low. Low molecular weight polymer and oligomers are, expectedly, not adsorbed because of their too small size which unables them to create a sufficient number of contact to ensure permanent adhesion; heat of adsorption in this case is too low to overcome thermal agitation, kT.

High MW's molecules do not adsorb either, presumably, because of their failure under our experimental conditions to wind around the nanotube, contradicting modeling predictions [5] or, most likely, because its incapacity to yield a sufficient number of adsorption contact-points to guarantee permanent adhesion. In order to do so a polymer chain should undergo a defolding process of its coiled conformation all along the tube axis. Such process is highly disadvantaged from an entropic viewpoint. Therefore, only an intermediate MW's chain can meet the compromise of being short enough to defold over the tube surface without too much entropic penalty but still able to create enough contact-points to offer an enthalpic gain high enough to link it permanently to the surface of the nanotube.

\section{Conclusions}

As many other new technologies, carbon nanotubes unique structure and characters have attracted excessively so much attention so fast that they can hardly live up to all our earliest expectations namely in polymer applications. The main reason hides in the fact that the way a long macromolecule come within reach of nano-sized solid differs fundamentally from the way it approaches conventional, relatively "unlimited" surface.

This is clearly demonstrated by the absence of adsorption of polybutadienes on CNT except through MW's narrow window in which the size of the macromolecule matches the size of the exposed surface. The consequence of such a lack in the establishment of the necessary interactions is to restrain adsorption and therefore, exclude the formation of a valuable interphase, which is responsible for the emergence of most of the reinforcement observable facts. This is evidenced by the non- effect of static deformation on dynamic modulus for CNT filled SSBR while CB filled one show first, a decrease then, an increase of the dynamic modulus upon deformation. The immobilized nature of the interphase is in this case presumably responsible for such a nonlinear deaging/physical aging behaviors.

\section{REFERENCES}

[1] K. Friedrich, S. Fakirov and Z. Zhang, "Polymer Composites: From Nano- to Macro-Scale,” Springer, 2005.

[2] B. Haida and T. L. Smith, "Dependance of Segmental Mobility in Polycarbonate on Time and Deformation," Polymer, Vol. 31, 1990, p. 1904.

[3] B. Haida, H. Deradji, A. Vidal and E. Papirer, "Polymers and Surfaces. A Versatil Combination in Recent Research Developments in Polymer Science,” In: H. Hommell, Ed., (Research Signpost, Trivandrum, India), 1990.

[4] V McBrierty and K. Packer, "Nuclear Magnetic Resonence in Solid Polymers,” Cambridge, 1993. http://dx.doi.org/10.1017/CBO9780511525278

[5] I. Gurevitch and S. Srebnik, "Conformational Behavior of Polymers Adsorbed on Wrapping of Nanotubes," Chemical Physics Letters, Vol. 444, 2007, p. 96. http://dx.doi.org/10.1016/j.cplett.2007.06.112 